\title{
3D PET Reconstruction with FORE and WLS-OS-EM
}

\author{
Charles W. Stearns, Member, IEEE, and Jeffrey A. Fessler, Senior Member, IEEE
}

\begin{abstract}
D PET reconstructions by the ordered subsets version of the ML-EM algorithm (typically referred to as "OS-EM") assume that the random- and scattered-corrected coincidence data retain their Poisson statistics. Negative projection values, which are possible after these subtractive corrections, are either truncated at zero counts or accommodated by adding an offset to the projection data, and to the corresponding forward-projected values, during the algorithm. These methods are effective due to the relatively low scatter and random fractions of most 2D PET acquisitions. In 3D PET data, particularly in acquisitions in the body, the scatter and random fractions are much higher. As a result, the OS-EM algorithm may not be the most appropriate for reconstruction for rebinned 3D PET data. We have implemented an ordered-subsets version of the weighted least-squares expectation maximization (WLS-EM) algorithm, or WLS-OS-EM, which is based on a Gaussian approximation to the statistics of the rebinned data. The weights used in the algorithm are based on the attenuation correction factors applied to the data. WLS-OSEM exhibits faster, and more consistent, convergence than MLOS-EM for projection data sets with substantial negative projection values created by subtractive data corrections.
\end{abstract}

\section{INTRODUCTION}

THE ordered-subsets version of the ML-EM algorithm [1], typically referred to as "OS-EM" but referred to as "ML-OS-EM" in this paper, is commonly used for clinical PET image reconstruction. 2D ML-OS-EM has also been used in conjunction with Fourier Rebinning (FORE) as an alternative to fully three-dimensional iterative reconstruction [2,3]. The ML-EM algorithm, and by extension ML-OS-EM as well, is predicated on the assumption that each data element represents a statistically independent Poisson random variable. While this is valid for raw coincidence data acquired by a PET scanner, each of the corrections applied to the data violates the assumption to some degree. Attenuation correction is of particular concern, because it multiplies the data by large factors ( 50 or more in the abdomen of large patients) that may vary greatly from data element to data element, distorting the data statistics severely. This problem can be alleviated by

Manuscript received November 13, 2002.

C. W. Stearns is with GE Medical Systems, Milwaukee, WI 53201 USA (telephone 262-312-7097, e-mail: charles.stearns@med.ge.com).

J. A. Fessler is with the Department of Electrical Engineering and Computer Science, University of Michigan, Ann Arbor, MI 48109 USA (telephone 734-763-1434, e-mail: fessler@umich.edu). incorporating the attenuation process into the iterative algorithm $[4,5]$.

The corrections for random and scattered coincidences are also problematic. This is because they are subtractive corrections, so there is a possibility that the data after correction will be negative. Since Poisson random variables are by definition non-negative, any negative values must be eliminated before ML-EM or ML-OS-EM can be attempted. Truncating the data at zero counts will certainly eliminate the negative values, but doing so risks biasing the data, and hence the reconstructed image, upwards. A better approach is to add an offset to the projection data equal in magnitude to the most negative data element, and add the same offset to the forwardprojected values during execution of the algorithm [6]. While this "shifted Poisson" technique makes the algorithm executable, large shift values will distort the data statistics and reduce the noise benefits of using iterative reconstruction. Since 3D PET acquisitions, particularly in the body, are characterized by high random and scattered coincidence fractions, the incidence of negative data values, and hence their consequences, may be expected to be greater in $3 \mathrm{D}$ than $2 \mathrm{D}$ data.

In our initial experience with FORE+ML-OS-EM with the shifted Poisson technique we experienced difficulty in achieving consistent data convergence. This is demonstrated in Fig. 1. Each image in the figure is a transaxial slice from an image set reconstructed from data acquired on a GE Advance PET scanner. The data was reconstructed using FORE (the sinograms were interpolated from 336 to 320 rows so that an efficient $5 \times 2^{n}$ FFT algorithm could be applied) and ML-OSEM using 2 iterations and 20 subsets. These ML-OS-EM parameters nearly match the 2 iterations and 28 subsets typically used in clinical 2D PET reconstructions on the Advance. The image of the NEMA NU-2 1994 scatter correction phantom (a $20 \mathrm{~cm}$ flood phantom with a $5 \mathrm{~cm}$ waterfilled cold spot), shown in the left panel of the figure, shows excellent convergence. The center and right panels, a Derenzo hot spot phantom and a uniform slice of the IEC body phantom, respectively, do not. If the reconstructions of these phantoms are continued to 8 iterations, good convergence is achieved, as shown in Fig. 2. Slow convergence of FORE+ML-OS-EM reconstructions have also been reported by Liu, et al. [3]. 
Clinical operation of a PET scanner requires consistent algorithm performance across a wide range of imaging conditions. Short reconstruction times, which can be interpreted for the iterative algorithms as meaning a low iteration count, are also preferred in routine use. Based on our initial experience, we concluded that an alternate formulation of the iterative reconstruction algorithm should be considered.

\section{WLS-EM AND WLS-OS-EM}

The WLS-EM algorithm seeks to minimize the least-squares cost function:

$$
f(x)=(y-G x)^{T} W(y-G x)=\sum_{i} W_{i}\left(y_{i}-[G x]_{i}\right)^{2},
$$

where $x$ represents the image; $y$, the rebinned projection data; $W$, a diagonal weighting matrix; and $G$, the projection matrix from image to data. The EM algorithm that minimizes this cost function is [7]:

$$
x^{n+1}=\left\lfloor x^{n}+D^{-1} G^{T} W\left(y-G x^{n}\right)\right\rfloor_{+},
$$

where $\left.L_{+}\right\rfloor_{+}$indicates a floor of zero on the image elements (i.e., clipping the negative values), and D is a diagonal matrix whose elements are defined as:

$$
d_{j}=\sum_{i} g_{i j} g_{i} w_{i}, \quad g_{i}=\sum_{j} g_{i j} .
$$

The ordered subsets version of the WLS-EM algorithm that we have implemented is described by the equation:

$$
x^{n, s+1}=\left\lfloor x^{n, s}+\alpha_{n} M D^{-1} G_{S}{ }^{T} W_{S}\left(y_{S}-G_{S} x^{n, s}\right)\right\rfloor_{+},
$$

where $x^{n, s}$ and $x^{n, s+1}$ represent the image before and after the application of the $s$ 'th subset, $\bullet_{s}$ denotes the portion of the data or projection matrix specific to the subset, $M$ is the number of subsets, and $\alpha_{n}$ is an term designed to insure convergence of the algorithm as the iterations proceed. If $\alpha_{n} \rightarrow 0$ as $n \rightarrow \infty$, $\Sigma_{n} \alpha_{\mathrm{n}}=\infty$ and $\Sigma_{n} \alpha_{\mathrm{n}}{ }^{2}<\infty$, then convergence is guaranteed [8]; the choice $\alpha_{\mathrm{n}}=k /(k+n)$ is an appropriate form for the relaxation term.

\section{ALGORITHM IMPLEMENTATION}

The algorithm is implemented in the following steps:

1. Backproject the image $x^{n, s}$ along the lines corresponding to the $s$ 'th subset,

2. Subtract the backprojected data from the input data,

3. Apply the weights to this difference data.

4. Forward project the weighted difference data.
5. Scale each pixel by $\alpha_{n} M d_{j}$ (note that $M d_{j}$ this can be precomputed),

6. Add this to the image $x^{n, s}$ to produce the updated image $x^{n, s+1}$.

7. Clip any negative values in $x^{n, s+1}$ to apply the nonnegativity constraint.

This algorithm is comparable in complexity, and therefore computation time, to the ML-OS-EM algorithm. The most striking difference between the two algorithms is that ML-OSEM uses division to compare the projected image to the data and multiplication to update the image in each subiteration, while WLS-OS-EM uses subtraction and addition, respectively. Each of these steps is comparable in complexity, and in both cases the processing time per iteration is dominated by the forward and backprojection steps.

The weights $w_{i}$ we use in the algorithm are based on the attenuation correction factors corresponding to each ray in the rebinned data:

$$
w_{i}=a_{i}^{2}
$$

where $a_{i}$ is defined as the ratio of attenuated to nonattenuated coincidence counts (i.e., $a_{i}$ is a number less than or equal to 1.0). These weights produce an algorithm which is identical to an unweighted least squares algorithm operating on data without attenuation correction (this is demonstrated in the Appendix), and therefore is a direct correlate to "attenuation weighted" ML-OS-EM algorithms. While different weighting schemes are possible, and may the subject of future investigation, the weights described in Eq. 5 have provided satisfactory results to date.

\section{RESUlts}

We performed a simulation study to explore the convergence of the WLS-OS-EM algorithm at a low iteration count. We simulated 2D projection data sets of an elliptical object with several hot and cold features at count levels corresponding to $100 \mathrm{~K}, 1 \mathrm{M}$ and $10 \mathrm{M}$ counts, with varying amounts of additive "background" noise (representing scattered and random coincidences) ranging from $0 \%$ to $300 \%$ of the true counts. An unbiased but noisy estimate of the background was subtracted from each sinogram to emulate the correction process, and the images were reconstructed using two iterations and 20 subsets of ML-OS-EM and WLS-OS-EM. The sinograms generated for this experiment are shown in Fig. 3, and the resulting images from MLS-OS-EM and WLS-OS-EM are shown in Fig. 4. Each algorithm produces an artifact-free reconstruction of the $10 \mathrm{M}$ count images, even in the presence of substantial levels of background noise. At lower total count levels, however, the ML images exhibit a significant artifact at the lateral edges of the phantom, and appear smoother, suggesting that they are not sufficiently converged. This artifact is not present in the corresponding WLS images. 
Fig. 5 shows the results of applying FORE+WLS-OS-EM to the phantom data presented in Figs. 1 and 2. Each phantom exhibits good convergence with 2 iterations and 20 subsets.

\section{CONCLUSION}

We have observed that WLS-OS-EM exhibits faster, and more consistent, convergence than ML-OS-EM with the shifted Poisson model for projection data sets with substantial negative projection values created by subtractive data corrections. 3D reconstruction using FORE+WLS-OS-EM has been implemented for the GE Advance PET scanner [9].

\section{APPENDIX}

We demonstrate here that WLS-EM with attenuation weights as defined in Eq. 5 is equivalent to an unweighted least squares EM algorithm applied to data without attenuation correction (denoted hereafter as "the alternate algorithm"). The extension of this demonstration to the ordered subsets algorithm is straightforward.

Consider the WLS-EM algorithm as described in Eq. 2, using weights as described in Eq. 5. The algorithm is defined by the equation:

$$
x^{n+1}=\left\lfloor x^{n}+D^{-1} G^{\prime T} A^{2}\left(y-G^{\prime} x^{n}\right)\right\rfloor_{+},
$$

where $G^{\prime}$ is used to designate the projection matrix for the system without compensation for attenuation, and $A$ is a diagonal matrix holding the attenuation factors $a_{i}$. The alternate algorithm is formed if we substitute attenuated data $(A y)$ a projection matrix that accounts for attenuation $\left(A G^{\prime}\right)$ and unity weights into Eq. 2, forming:

$$
x^{n+1}=\left\lfloor x^{n}+D^{-1}\left(A G^{\prime}\right)^{T}\left(A y-A G^{\prime} x^{n}\right)\right\rfloor_{+},
$$

It is clear that these two algorithms are identical if the value of $D$ is the same in both equations.

The elements of matrix $D$ are defined by Eq. 3. Using the attenuation weights, these elements are computed as:

$$
d_{j}=\sum_{i} a_{i}^{2} g_{i j}^{\prime}\left(\sum_{j} g_{i j}^{\prime}\right)
$$

In the alternate algorithm,

$$
g_{i j}=\left(A G^{\prime}\right)_{i j}=a_{i} g_{i j}^{\prime}
$$

Substituting this into Eq. 3,

$$
d_{j}=\sum_{i} a_{i} g_{i j}^{\prime}\left(\sum_{j} a_{i} g_{i j}^{\prime}\right)
$$

We can factor $a_{i}$ out of the inner sum, reconciling Eq. 10 with Eq. 8 and establishing the equivalence of the two algorithms.

\section{REFERENCES}

[1] H. M. Hudson, and R. S. Larkin, "Accelerated image reconstruction using ordered subsets of projection data," IEEE Trans. Med. Imag., vol. 13, no. 4, pp. 601-609, Dec. 1994.

[2] P. E. Kinahan, C. Michel, M. Defrise, D. W. Townsend, M. Sibomana, M. Lonneux, D. F. Newport, and J. D. Luketich, "Fast iterative image reconstruction of 3D PET data," 1996 IEEE Nuclear Science Symposium Conference Record, pp. 1918-1922, Nov. 1996.

[3] X. Liu, C. Comtat, C. Michel, P. Kinahan, M. Defrise, and D. Townsend, "Comparison of 3-D reconstruction with 3D-OSEM and with FORE+OSEM for PET," IEEE Trans. Med. Imag., vol. 20, no. 8, pp. 804-814, Aug. 2001.

[4] E. U. Mumcuoglu, R. Leahy and S. R. Cherry, "Bayesian reconstruction of PET images: Methodology and performance analysis," Phys. Med. Biol., vol. 41, pp. 1777-1807, 1996.

[5] C. Comtat, P. E. Kinahan, M. Defrise, C. Michel, D. W. Townsend, "Fast reconstruction of 3D PET data with accurate statistical modeling," IEEE Trans. Nucl. Sci., vol. 45, no. 3, pp. 1083-1089, June 1998.

[6] M. Yavuz, and J. A. Fessler, "Statistical image reconstruction methods for randoms-precorrected PET scans," Med. Im. Anal., vol. 2, no. 4, pp. 369-378, Dec. 1998.

[7] J. A. Fessler, and A. O. Hero, "Space-alternating generalized expectation-maximization algorithm" IEEE Trans Signal Processing, vol. 42, no. 10, pp. 2664-2677, Oct. 1994.

[8] S. Ahn, and J. A. Fessler, "Globally convergent ordered subsets algorithms: application to tomography," 2001 IEEE Nuclear Science Symposium Conference Record, paper M01-2, Nov. 2001.

[9] S. D. Wollenweber, "Analysis of the parameterization of FORE-WLS reconstruction in 3D whole-body PET imaging," 2002 IEEE Nuclear Science Symposium/ Medical Imaging Conference, paper M10-99, Nov. 2002.
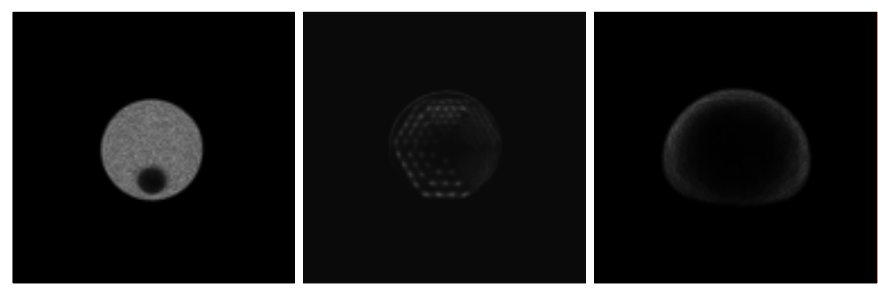

Figure 1. FORE+ML-OS-EM reconstructions of data from GE Advance, using 2 iterations and 20 subseets.
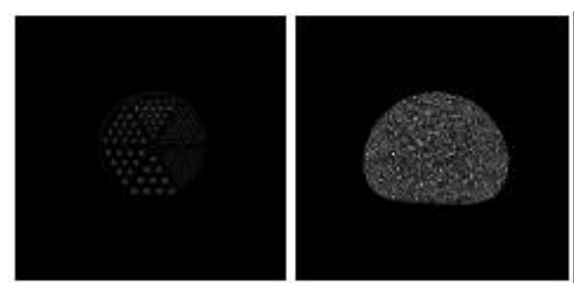

Figure 2. Reconstructions of the Derenzo and IEC phantoms from Fig. 1, using 8 iterations and 20 subsets. 


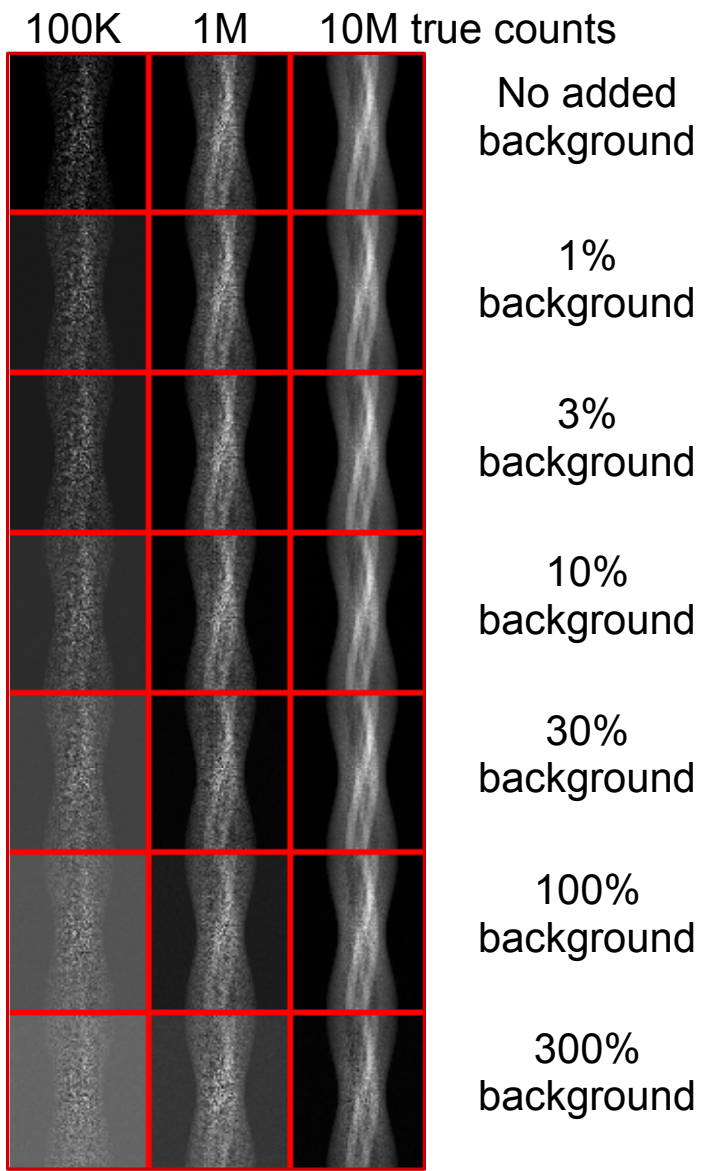

Figure 3. Sinograms used to investigate convergence properties of ML-OS-EM and WLS-OS-EM algorithms.

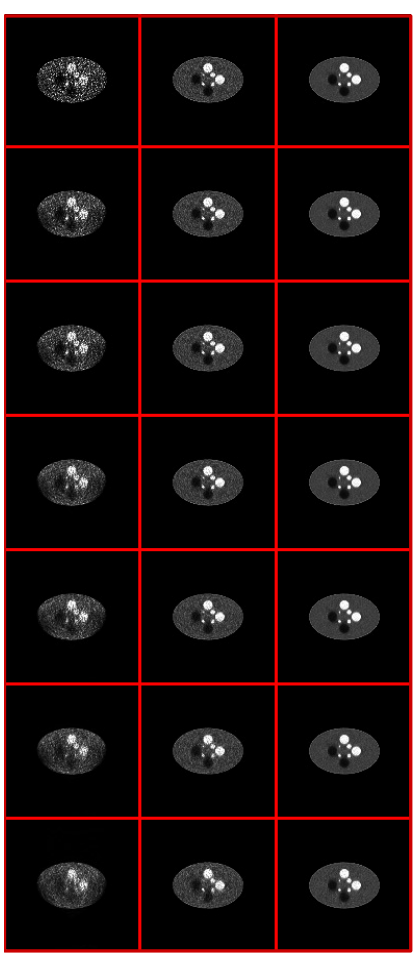

ML-OS-EM

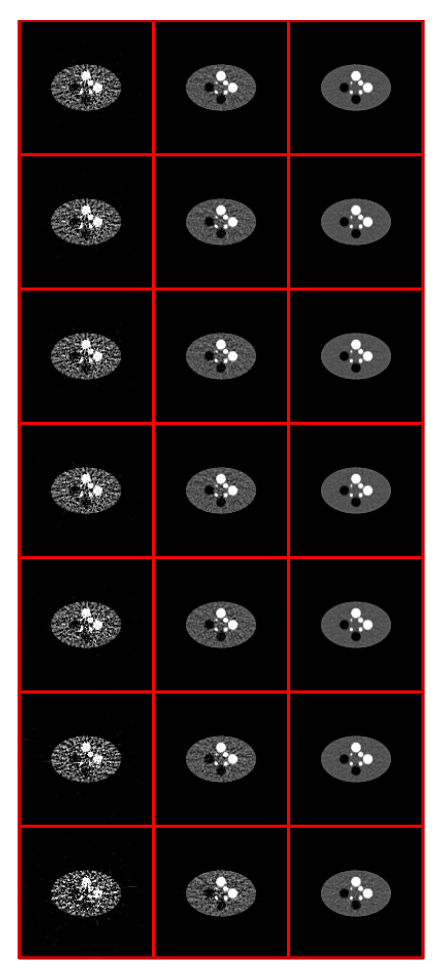

WLS-OS-EM
Figure 4. Reconstructed images of the sinograms in Fig. 3 using ML-OS-EM and WLS-OS-EM.
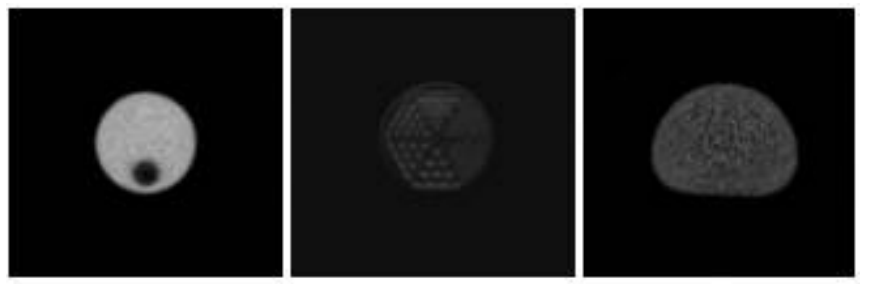

Figure 5. FORE+WLS-OS-EM reconstructions of data from GE Advance, using 2 iterations and 20 subseets. 